\title{
Modeling Flow around Bluff Bodies and Predicting Urban Dispersion Using Large Eddy Simulation
}

\author{
YU - HENG TSENG, ${ }^{\dagger, \$}$ \\ CHARLES MENEVEAU,$^{\dagger}$ AND \\ MARC B. PARLANGE*, ;
}

Department of Mechanical Engineering, Center for Environmental and Applied Fluid Mechanics, and

Department of Geography and Environmental Engineering, The Johns Hopkins University, 3400 North Charles Street, Baltimore, Maryland 21218, and School of Architecture, Civil and Environmental Engineering, Ecole Polytechnique Fédérale de Lausanne, (EPFL) CH-1015 Lausanne, Switzerland

Modeling air pollutant transport and dispersion in urban environments is especially challenging due to complex ground topography. In this study, we describe a large eddy simulation (LES) tool including a new dynamic subgrid closure and boundary treatment to model urban dispersion problems. The numerical model is developed, validated, and extended to a realistic urban layout. In such applications fairly coarse grids must be used in which each building can be represented using relatively few grid-points only. By carrying out LES of flow around a square cylinder and of flow over surface-mounted cubes, the coarsest resolution required to resolve the bluff body's cross section while still producing meaningful results is established. Specifically, we perform grid refinement studies showing that at least 6-8 grid points across the bluff body are required for reasonable results. The performance of several subgrid models is also compared. Although effects of the subgrid models on the mean flow are found to be small, dynamic Lagrangian models give a physically more realistic subgridscale (SGS) viscosity field. When scale-dependence is taken into consideration, these models lead to more realistic resolved fluctuating velocities and spectra. These results set the minimum grid resolution and subgrid model requirements needed to apply LES in simulations of neutral atmospheric boundary layer flow and scalar transport over a realistic urban geometry. The results also illustrate the advantages of LES over traditional modeling approaches, particularly its ability to take into account the complex boundary details and the unsteady nature of atmospheric boundary layer flow. Thus LES can be used to evaluate probabilities of extreme events (such as probabilities of exceeding threshold pollutant concentrations). Some

\footnotetext{
* Corresponding author e-mail: Marc.Parlange@epfl.ch.

${ }^{\dagger}$ Department of Mechanical Engineering and Center for Environmental and Applied Fluid Mechanics, The Johns Hopkins University.

₹ Ecole Polytechnique Fédérale de Lausanne and Department of Geography and Environmental Engineering, The Johns Hopkins University.

$\$$ Present address: One Cyclotron Rd, MS:50F-1650, Computational Research Division, Lawrence Berkeley National Laboratory, Berkeley, CA 94720.
}

comments about computer resources required for LES are also included.

\section{Introduction}

There is a growing concern about air pollution in urban environments. To assess the magnitude of the problem, accurate prediction of the transport and dispersion of airborne contaminants in urban environments is needed. However, it is difficult to simulate such physical processes accurately due to complex surface topography. An urban canopy consists of a large collection of buildings, trees, and other obstacles that are aggregated into complex structures. When this rough surface interacts with the atmospheric flow above it, the disturbed flow field can become extremely complex depending on the obstacles' geometry, spacing, and the spatial arrangement. Wind-tunnel experiments have confirmed that small-scale building features can significantly influence transport and dispersion $(1,2)$. The complex flow fields directly affect transport of released substances. Whereas some aggregate effects of heterogeneous surfaces can be parametrized using averaging assumptions (3-5), more accurate results can only be obtained by explicitly describing the complex boundary condition formed by the bluff bodies (such as buildings). This is possible in principle in Large Eddy Simulation (LES) where large-scale eddies and their unsteady time-evolution are captured explicitly, while smallscale eddies are described using subgrid-scale (SGS) models. Application of LES in urban environments using implicit methods (relying on numerics to mimic turbulence small scales) is also being pursued (6).

LES that covers horizontal domains of $1 \mathrm{~km}$, including a number of buildings about $50 \mathrm{~m}$ wide, typically uses a total of 100-200 grid-points in each direction. This means that the number of grid-points available to cover the horizontal extent of a single building is at most ten. This resolution is marginal and prompts one to question the overall accuracy of LES simulation in these situations. Hence, as part of the development of an LES model to simulate the flow over an urban canopy, we undertake the task of validating the LES model in simple flow around square bluff bodies for which detailed experimental data are available for comparison. Specifically, we consider flow around a square cylinder and flow over a matrix of surface-mounted cubes, using data obtained from the ERCOFTAC (European Research Community on Flow, Turbulence and Combustion) database (7, 8). Both flow configurations are important benchmark tests because many factors are involved, including stagnationpoint flow, flow separation, streamline curvature, recirculation, vortex shedding, and chaotic three-dimensional wake structure at increasing Reynolds number. Detailed comparisons between LES and data in these flows have been already performed $(9,10)$. However, based on the needs of LES of urban canopy flows, our focus here is on marginal numerical resolutions. Several different eddy-viscosity subgrid-scale models are tested to quantify the effects of SGS models in these fairly coarse-resolution LES. The focus in this paper is on the flow and velocity field characteristics under neutral atmospheric conditions, including transport of scalar contaminants. The applicability of the LES tool is then illustrated in simulations of neutral atmospheric boundary-layer flow and scalar transport over a realistic urban geometry.

This paper is organized as follows. Section 2 briefly describes the numerical methods and the SGS models. We

VOL. 40, NO. 8, 2006 / ENVIRONMENTAL SCIENCE \& TECHNOLOGY - 2653 
use a pseudospectral numerical scheme in the horizontal planes and a centered, second-order scheme in the vertical direction. Objects in the flow are described using the Immersed Boundary Method (IBM) (11). To mitigate problems associated with coupling spectral methods and IBM, we extend the idea of generalized ghost cell $\operatorname{IBM}(11,12)$ to filter out high-frequency modes while preserving the spectral numerical accuracy in the core of the flow away from the boundaries.

In addition, three SGS models are assessed in the present study. The first is the classic eddy-viscosity Smagorinsky model $(13,14)$. The second model tested is the dynamic SGS model (15) that makes use of the hypothesis of scaleinvariance (see ref 16) and determines the coefficient from suitable analysis of the large scales during the simulation. For complex geometries, we use its Lagrangian version (17). The third SGS model is the dynamic, scale-dependent Lagrangian model which allows deviations from strict scaleinvariance (18). As shown in refs 18 and 19, atmospheric boundary layer flows require the use of scale-dependent dynamic models to accurately represent the log-law in flow over flat surfaces of homogeneous or heterogeneous surface roughness distributions. Our main objective is to assess the results of the scale-dependent dynamic model when bluff bodies are added to the simulation domain.

Section 3 applies the numerical code using flow around a square cylinder and investigates the effects of SGS models and grid resolution. Section 4 quantifies the effects of various SGS models in turbulent flow over a matrix of surfacemounted cubes. In section 5, we present a realistic application of flow over downtown Baltimore, MD.

\section{Description of the Numerical Simulations}

Numerical integration of the filtered incompressible NavierStokes equations (see eq 1 below) is used to simulate threedimensional, neutral, turbulent flow

$$
\frac{\partial \bar{u}_{j}}{\partial x_{j}}=0, \frac{\partial \bar{u}_{i}}{\partial t}+u_{j}\left(\frac{\partial \bar{u}_{i}}{\partial x_{j}}-\frac{\partial \bar{u}_{j}}{\partial x_{i}}\right)=-\frac{\partial \bar{p}^{*}}{\partial x_{i}}+\bar{F}_{i}+f_{\mathrm{i}}-\frac{\partial \tau_{i j}}{\partial x_{j}}
$$

where $\bar{u}_{j}$ is the resolved velocity vector, and the rotational form of the equation is used for kinetic energy and mass conservation (20). Buoyancy and stratification effects are not included in the present study. The external forcing $\left(\bar{F}_{i}\right)$ includes a mean streamwise pressure forcing. Additional $f_{i}$ is a so-called immersed boundary forcing used to simulate the effects of obstacles in the flow. Stress $\tau_{i j}$ is the deviatoric part of the SGS stress tensor (see review in ref 21). It represents the effect of small eddies that are smaller than the resolved scales of motion. Note that the viscous term is neglected in our simulation due to the high Reynolds number and the effect of the wall layer (the viscous sublayer is not resolved) (22). The effective kinematic pressure, $\bar{p}^{*}$, is formulated so that the velocity field remains divergence free. Taking the divergence of the momentum equation and using the continuity equation results in a Poisson equation for pressure that can be solved for $\bar{p}^{*}$.

In LES the larger scales of turbulent flow are resolved at the grid scale, while the smaller, subgrid scales are parametrized. The success of LES depends on how accurately the SGS stresses are modeled. The effects are more noticeable and crucial when fairly coarse grids are used. Three subgrid scale models are evaluated in this study. They are all variations of the SGS parametrization most often used in LES, the Smagorinsky model (13). It expresses the SGS flux as a function of the resolved strain rate tensor $\bar{S}_{i j}=\left(\partial_{j} \bar{u}_{i}+\partial_{i} \bar{u}_{j}\right) / 2$ using

$$
\tau_{i j}=-2 v_{\mathrm{sgs}} \bar{S}_{i j}=-2\left(c_{\mathrm{s}, \Delta} \Delta\right)^{2}|\bar{S}| \bar{S}_{i j}
$$

where $\Delta$ is the filter width (or mesh-size) and $v_{\text {sgs }}$ is the SGS viscosity. The model uses the viscous analogy and the mixing length approach. The only unknown in eq 2 is the Smagorinsky coefficient $c_{\mathrm{s}, \Delta}$. The three models tested in this work differ only in how $c_{\mathrm{s}, \Delta}$ is specified.

The first, most traditional approach is to use a constant coefficient (e.g. 0.16) estimated from the theory of isotropic homogeneous turbulence (see review in ref 16). In the present tests of the classic Smagorinsky model, the Smagorinsky constant is set to $c_{\mathrm{s}, \Delta}=0.16$. The model is designated SM in the tests below.

It is known that the classic Smagorinsky model fails when the filter-scale approaches the limits of the inertial range, such as in the vicinity of solid boundaries. The Smagorinsky coefficient must decrease close to the surface, and attempts to impose empirical wall damping functions have shown that the model is overdissipative regardless of the damping function (23).

The dynamic model that was developed by ref 15 and modified by ref 24 dispenses with ad-hoc damping functions. Instead, it determines the coefficient $c_{\mathrm{s}, \Delta}$ dynamically from the resolved turbulence during the simulation. It makes the major assumption of scale-invariance in the sense that the coefficient deduced from the large resolved scales is used in the model of the small scales. One major problem of the dynamic model is that if the model coefficient is determined locally, it typically displays very large oscillations causing numerical instability associated with possible negative values. Thus averaging is needed for the model to be acceptable in terms of performance (15). Typically, the required statistical averaging is performed over a spatially homogeneous direction (e.g. horizontal plane). This requires spatial homogeneity in the flow domain. However, it is difficult to define spatial homogeneity in complex three-dimensional turbulent flows over bluff bodies. A better approach is to perform averaging in time following fluid pathlines. This Lagrangian model (17) is well suited for applications with complex geometries because it preserves both local variability and Galilean invariance and does not require spatial homogeneity. For detailed descriptions of this so-called scale-invariant Lagrangian dynamic model (SILM), see refs 17 and 18. We use the Gaussian filter for test filtering in this study because it is compact in both physical space and wavenumber space, as opposed to a spectral cut-off filter that would introduce numerical errors associated with strong Gibbs oscillations near the bluff body.

The third subgrid model tested in the present study is the one which relaxes the assumption that the coefficient is scaleinvariant. Scale-invariance does not always hold, typically near solid walls where the filter scale and the integral scale of the turbulence are both on the order of the distance to the wall. To account for scale effects, we use a fully dynamic formulation in which the scale-dependence is derived from the resolved scales through an additional test-filtering operation. This approach has been successfully implemented with the planar-averaged dynamic approach for ABL flows by ref 19 and another simplified variant using the Lagrangian averaging approach by ref 18 . The main assumption is a power-law behavior for the scale dependence, i.e., $c_{\mathrm{s}, \Delta}=c_{\mathrm{s}, \alpha \Delta}(\Delta / \alpha \Delta)^{\phi}$. Consequently, the scale-dependence parameter $\beta$ (the ratio of coefficients at scales $\alpha \Delta$ and $\Delta$ ) is equal to $\beta$ evaluated between scales $\alpha^{2} \Delta$ and $\alpha \Delta$ (i.e. the power-law assumption is equivalent to assuming $\beta$ is scaleinvariant (18)). From the locally known values of $c_{\mathrm{s}, 2 \Delta}$ and $c_{\mathrm{s}, 4 \Delta}, \beta$ can be written as

$$
\beta=c_{\mathrm{s}, 4 \Delta}^{2} / c_{\mathrm{s}, 2 \Delta}^{2}=c_{\mathrm{s}, 2 \Delta}^{2} / c_{\mathrm{s}, \Delta}^{2}
$$

Then the coefficient at scale $\Delta$ is obtained immediately, $c_{\mathrm{s}, \Delta}^{2}$ 


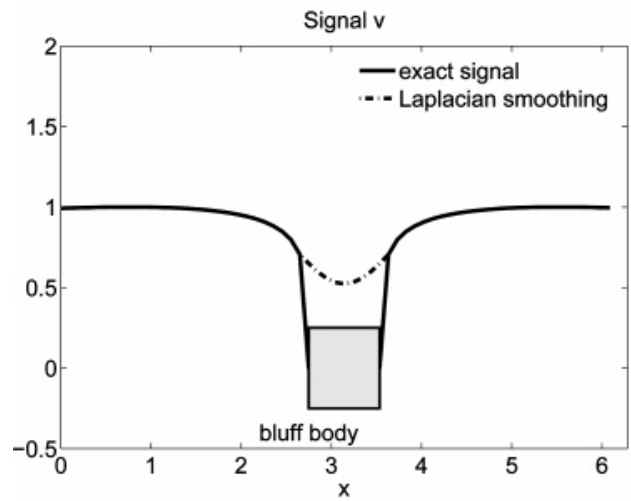

(a)

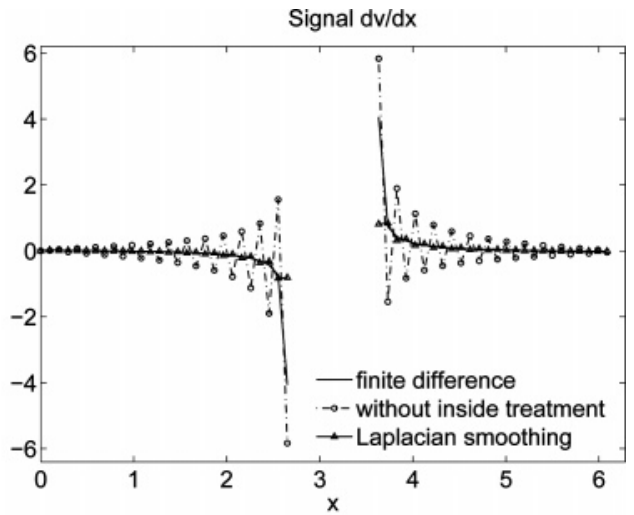

(b)

FIGURE 1. Illustration of Gibbs phenomenon and its reduction using different methods. (a) Sample one-dimensional signal $u(x)$. It is constructed to resemble a uniform flow as it passes across a bluff body. The velocity near the bluff body boundary is approximated by a logarithmic profile. (b) The derivative $\mathrm{d} v / \mathrm{d} x$ of the signal in the front part of the test case, as evaluated using different methods.

$=c_{\mathrm{s}, 2 \Delta}^{2} / \beta$. For more details about the scale-dependent Lagrangian dynamic model (denoted as SDLM), see ref 18.

The JHU-LES code uses a pseudospectral approach in the horizontal directions and a second-order accurate centered difference scheme in the vertical direction. The centered difference scheme necessitates the use of a staggered grid in the vertical direction. The second-order AdamsBashforth scheme is used for time advancement. Full dealiasing of the convective terms is achieved by padding and truncation. More detailed discussion of the code can be found in refs 25 and 19 .

The presence of bluff bodies in the flow is modeled using the IBM (11). The IBM specifies a body force $\left(f_{i}\right)$ in such a way as to simulate the presence of a surface without altering the computational grid. Using the IBM, bodies of almost arbitrary shape (but with features larger than grid scale) can be dealt with, and different types of boundary conditions can be implemented. Furthermore, flows with multiple buildings may be computed at reasonable computational cost. The immersed boundary forcing $f_{i}$ is zero in the fluid and nonzero inside the bluff bodies. This forces the velocity field to zero in the bluff body $\left(\Lambda_{0}\right)$ effectively representing the presence of objects.

Using IBM to represent sharp bluff bodies may cause problems in the pseudospectral method. It is well-known that when using Fourier representation the Gibbs phenomenon commonly occurs at a point of discontinuity. The sharp discontinuity introduces high-frequency oscillation. To minimize the effects of Gibbs phenomenon around the bluff bodies, the velocity field inside the building is modified by adding a ghost-velocity function $\left(v_{\mathrm{d}}\right)$ before taking the derivative (26), i.e., $v_{\mathrm{d}}(\mathbf{x})=v_{\mathrm{g}}(\mathbf{x})$ if $\mathbf{x} \in \Lambda_{0}$ (bluff bodies), zeros otherwise. In each pseudospectral horizontal plane (fixed height), the ghost-velocity function is constructed by applying a Laplacian smoothing operator iteratively to the updated velocity field $v_{\mathrm{g}}(i, j)$ inside the bluff bodies (for any location $\mathbf{x}(i, j)$, and where $(i, j)$ are the position indices in the plane) such that

$$
\begin{array}{r}
v_{\mathrm{g}}^{\text {new }}(i, j)=(1-4 \lambda) v_{\mathrm{g}}^{\text {old }}(i, j)+\lambda\left[\nu_{\mathrm{i}}^{\text {old }}(i+1, j)+v_{\mathrm{g}}^{\text {old }}(i-\right. \\
\left.1, j)+v_{\mathrm{i}}^{\text {old }}(i, j-1)+v_{\mathrm{g}}^{\text {old }}(i, j+1)\right]
\end{array}
$$

where $\lambda=0.24$ is the weight used in this study. The value is chosen to preserve more weight on the neighboring points and less weight on the interpolated points. The same value is used in the various successful tests shown in the present paper, and thus we believe this may represent a fairly universally applicable value. The smoothing of the velocity field is initialized from the velocity at the end of the previous time step and is only used during differentiation operations. The approach resembles the reconstruction scheme in refs 26 and 27. Adding the ghost-velocity function using the Laplacian operator inside the bluff bodies acts as a low-pass filter to remove the unphysical high-frequency oscillations introduced by the discontinuity without affecting the outer flow field.

The method is illustrated on a one-dimensional signal (Figure 1). The original signal $v(x)$ resembles the streamwise velocity along the centerline in a uniform flow passing around a bluff body. Near the bluff body boundary, the velocity decreases very rapidly to zero, but at the first grid-point away from the boundary, the velocity is still large. The unmodified velocity inside the object is zero, and we observe significant oscillation near the boundary when directly taking the spectral derivative of the signal across the bluff body (displayed as circles $-{ }^{\circ}$ in Figure 1(b)). Adding the ghostvelocity function inside the bluff body removes the overshoot in $\mathrm{d} v / \mathrm{d} x$ significantly (Figure 1(b)). We also observed significant improvement in several two-dimensional tests. The vertical gradients obtained using this method are then used when calculating pressure using the Poisson equation.

The code uses zero normal velocity and logarithmic type boundary condition for the tangential stresses at both the bottom boundary and the bluff body surface, e.g. refs 28,21 , and 18 . The tangential stress condition uses the assumption that the first grid point around the bluff body is far outside the laminar sublayer. More realistic boundary conditions for high Reynolds number flows, such as used in Detached Eddy Simulation (DES, see refs 29 and 30), require relatively fine discretizations near the wall and also require an extra transport equation for the eddy-viscosity which has not yet been tested extensively in conjunction with the dynamic model. The logarithmic boundary condition around the bluff bodies is chosen here due to its simplicity and its good performance in our extensive tests in conjunction with the scale-dependent dynamic model $(19,5)$.

The pseudospectral method assumes periodic boundary conditions with laminar inflow. The bluff body's wake is thus fed into the domain upstream of the bluff body, using a relaxation zone that linearly brings the velocity to the desired laminar inflow. The zone is located between $x=(1 / 24) L$ and $x=(1 / 16) L$, where $L$ is the domain size. The inflow velocity $u_{\mathrm{o}}$ is specified at $x=(1 / 16) L$. Let $u_{\mathrm{b}}$ be the actual velocity arising from the periodic boundary condition at $x=(1 / 24) L$. In the relaxation zone, a force is added to the momentum equation to bring the velocity to a relaxation velocity $u_{\mathrm{r}}=$ $(1-r) u_{\mathrm{o}}+r u_{\mathrm{b}}$, where $r$ is the distance ratio, $r=3-48 x / L$, 


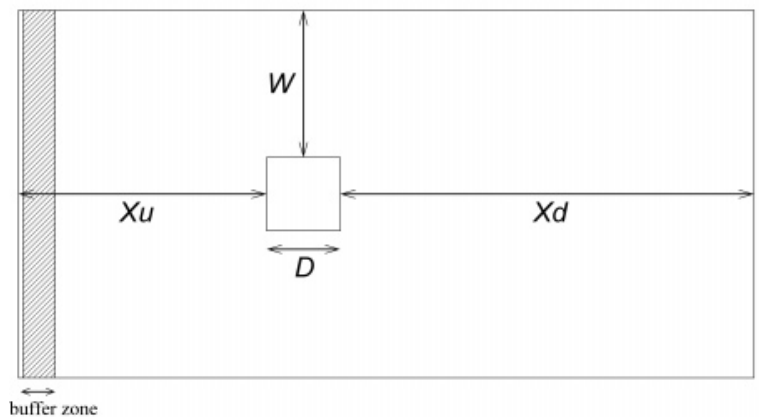

(a)

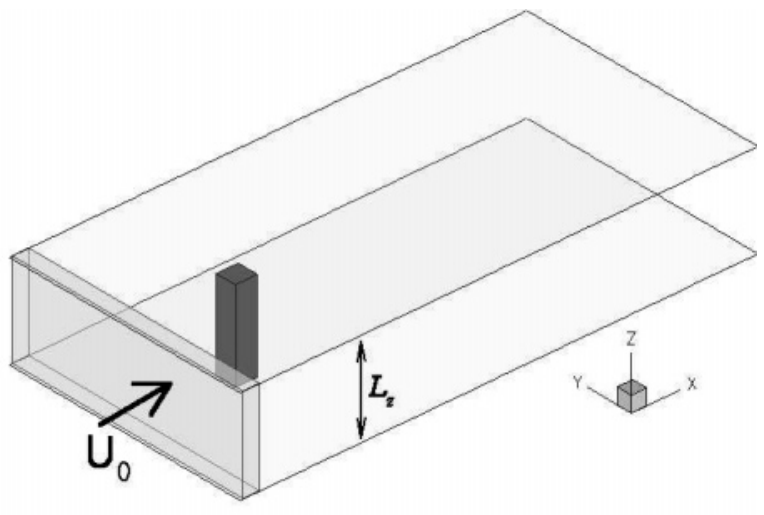

(b)

FIGURE 2. Computational domain of flow around a square cylinder. The shaded regions represent the buffer zone which relaxes the velocity toward the uniform inflow condition.

between the relaxation velocity location and the inflow location.

Pollutant transport and dispersion is modeled by an additional filtered scalar transport equation

$$
\frac{\partial \bar{C}}{\partial t}+\frac{\partial\left(\bar{u}_{j} \bar{C}\right)}{\partial x_{j}}=\frac{\partial}{\partial x_{j}}\left(v_{\mathrm{sgs}} S c_{\mathrm{sgs}}^{-1} \frac{\partial \bar{C}}{\partial x_{\mathrm{j}}}\right)
$$

where $C$ denotes the concentration of pollutant (in units of mass per unit volume). The turbulent subgrid-scale viscosity, $v_{\text {sgs, }}$, is dynamically calculated using the scale-dependent Lagrangian model described above in the context of the momentum equation. $S c_{\mathrm{sgs}}$ is the turbulent subgrid-scale Schmidt number that governs the relative strength of the diffusivity of the scalar field. Several approaches exist in specifying $S c_{\text {sgs. }}$. The traditional approach is to set it to a constant. From physical arguments, a value often chosen is $S c_{\mathrm{sgs}} \sim O(1)$, as unresolved turbulent eddies are known to transport scalars with approximately similar effectiveness than they transport momentum. A fundamentally more rigorous approach is to employ the dynamic procedure to determine the Schmidt number from the large scales. Recently, a scale-dependent dynamic version of such a model has been developed (31). However, for complex geometries such as urban canopies, it must also be implemented with Lagrangian averaging since no direction of statistical homogeneity exists. Such a model for scalar diffusivity adds considerably to the complexity of the code and has not yet been implemented and tested in complex-geometry flows such as urban canopy flows. Another often used approach is to rely primarily on "numerical diffusion" by employing a numerical scheme that explicitly preserves "monotonicity" (32). The motivation for such approaches is that high-order numerical solution schemes for solving the conserved scalar transport equation without numerical diffusion may yield local values of the concentration that become negative or exceed the maximum concentration injected into the domain. Such trends are unphysical and can lead to numerical instabilities.

To limit computational complexity, we opt in this work to prescribe a (nondynamic) fixed value of $S c_{\text {sgs. }}$. Also, to separate clearly between effects from numerical discretization and physical modeling, we opt to maintain the high-order code instead of using monotonicity-preserving approaches. After some numerical experimentation, we have found that setting $S c_{\mathrm{sgs}}=0.05$ (i.e. using a scalar diffusivity 20 times as large as that of the momentum equations) avoids the unphysical fluctuations in the computed scalar fields. Values closer to unity yielded severe Gibbs phenomena near the building boundaries, due to the sudden changes in concentration there. As a consequence of using $S c<1$, scalar fluctuations are "smoothed" over length-scales significantly larger than the computational mesh-size. Future improvements to the model are needed to allow combining the Lagrangian dynamic model with the IBM and the spectral method, without incurring unphysical Gibbs oscillations. Our rationale for focusing on accuracy in determining the advective velocities while using a very approximate method to account for scalar diffusion is that, at high Reynolds numbers, the advection of scalars is the crucial element that will determine whether pollutants will enter and stay in recirculating regions, whether vortices will form, etc., whereas diffusion of the scalar only will determine how "smoothed out" the scalar concentration fields are.

\section{Three-Dimensional Flow around a Square Cylinder}

The first test case is flow around a square cylinder at a Reynolds number of $R e=22000$ (based on the square size and the inflow velocity). Reference data are from Laser Doppler Velocimetry (LDV) measurements, reported in refs 7 and 33. Data are available for the time-averaged and the phase-averaged flow along particular directions. A recent workshop was devoted to large eddy simulation of this test case (34), and some additional recent LES results are reported in refs $9,35,10$, and 36 .

Uniform inflow is prescribed, and a periodic boundary condition is used in the streamwise direction. The flow is then forced away from the periodic boundary inlet toward the required uniform inflow condition. This is done within a narrow buffer region upstream of the obstacle (Figure 2). Figure 2 shows the computational domain and the square cylinder. The characteristic dimensions are the following: $X_{\mathrm{u}} / D=5, X_{\mathrm{d}} / D=18, W / D=5.5$, and $L_{z} / D=6$. The boundary conditions are periodic on all lateral boundaries. Free-slip (zero stress) is applied at both the top and bottom, to minimize end effects and thus simulate a nominally infinitely long cylinder. A log-law boundary condition is applied at the square cylinder side walls.

The flow field is initialized with random perturbations and advanced in time until statistically steady-state conditions are established, also characterized by periodic shedding of large-scale vortices from the cylinder. The influence of spatial resolution on the results for all models is investigated. Details of these simulations are provided in Table 1 . The grid distribution is uniform. The coarsest grid is $96 \times 48 \times 24$ which includes 4 grid points spacing for dimension $D$ (across 


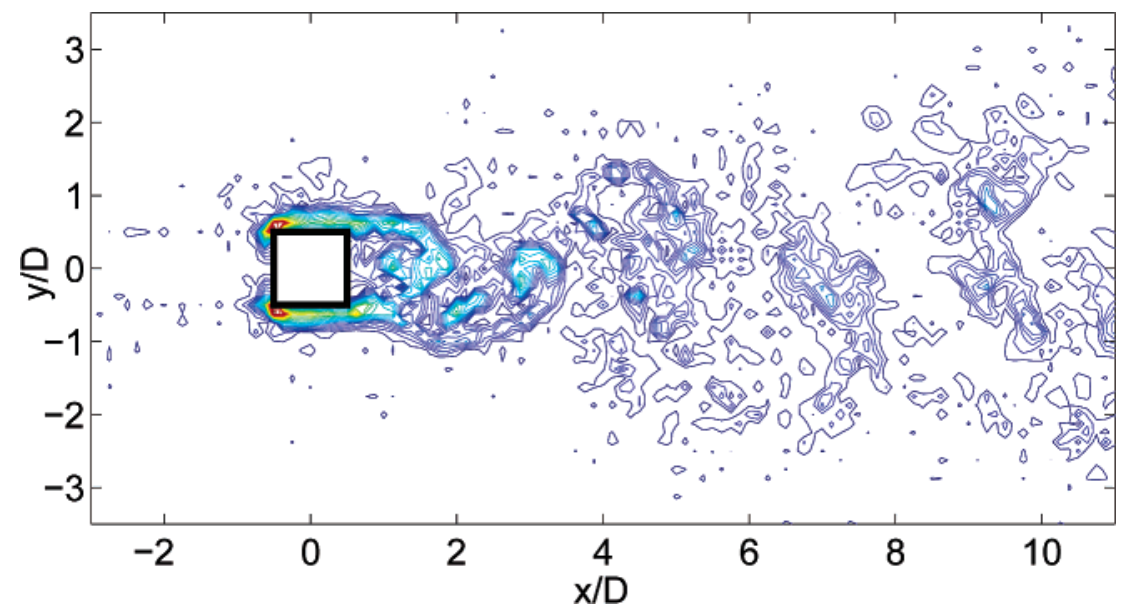

FIGURE 3. Instantaneous vorticity magnitude from $w D / U_{0}=0.4$ to $w D / U_{0}=10$ in the flow around a square cylinder.

\section{TABLE 1. Summary of the Simulations}

\begin{tabular}{cr} 
case & \multicolumn{1}{c}{ grid size } \\
G1 & $96 \times 48 \times 24$ \\
G2 & $144 \times 72 \times 24$ \\
G3 & $192 \times 96 \times 24$
\end{tabular}

${ }^{a} N_{x}$ : number of grid points spacing across the square cylinder.

the obstacle). Note that in this case the distance from the cylinder surface to the nearest grid point is $0.25 D$, clearly outside of the laminar sublayer and arguably even insufficient to capture gross features of the boundary layers around the body. Several simulations are performed with the different SGS models: Smagorinsky model (SM), scale-invariant Lagrangian model (SILM), and scale-dependent Lagrangian model (SDLM).

A good example of the effect of obstacles on the turbulent flow can be seen in Figure 3, which shows instantaneous contours of the spanwise vorticity magnitude with the SDLM on grid G3. An unsteady recirculation region and the development of the Kármán vortex street are clearly observed. The flow appears to be highly turbulent and threedimensional in the wake region. Note the presence of both large and small structures in the wake. The flow structures tend to increase in size with increasing downstream distance. The vortices arising from the instabilities of the shear layers mix in the primary Kármán vortices before propagating downstream.

As a first step, we compare the statistical results from different grid resolutions in order to address the main question of this paper: what is the minimum number of grid-points across the bluff body that will yield accurate results. The simulation results are analyzed to determine the predicted recirculation length $\left(L_{\mathrm{W}} / D\right)$ and the Strouhal number $(S t)$. The recirculation length is defined as the distance between the back of the square cylinder and the downstream location of zero mean velocity. Strouhal number $(S t)$ is determined from the peak in time-spectra. All results are compared with previous experimental and numerical results in Table 2 .

As can be seen, the recirculation length depends on both resolution and SGS models, while the Strouhal number is less sensitive to the SGS models. The comparison of the mean streamwise velocity using SDLM with different grid resolutions is shown in Figure 4(a) along the wake centerline $(y / D$ $=0$ ) and Figure 4(b) in the boundary layer at the center of the cylinder side $(x / D=0)$, respectively. The time-averaged quantities are calculated by averaging over about 20 vortex shedding cycles after an initial development time of 150D/

\begin{tabular}{|c|c|c|}
\hline & $L_{w} / D$ & $S t=f D / U_{0}$ \\
\hline experimental data (33) & 1.38 & $0.132 \pm 0.004$ \\
\hline simul & & 0.135 \\
\hline $\operatorname{sim} u$ & {$[1.29,1.34]$} & {$[0.129,0.135]$} \\
\hline ons TAMU2 (34) & {$[0.89,2.96]$} & {$[0.07,0.15]$} \\
\hline SDLM on G1 & 1.49 & 0.144 \\
\hline on $\mathrm{G} 2$ & 1.25 & 0.138 \\
\hline G3 & 1.36 & 0.141 \\
\hline & 1.4 & 0.140 \\
\hline SILM on G3 & 1.55 & 0.141 \\
\hline
\end{tabular}

$U_{0}$. Calculations with the coarse grid (G1) do not appear very satisfactory, but the calculations using medium resolution (G2 or G3) give reasonable results, except that the streamwise velocity in the wake is slightly underpredicted in G2 and G3. Figure 4(b) shows that none of the three resolutions is sufficient to reproduce the flow reversal associated with flow separation around the front corner. This is a typical situation encountered in practical LES of a complex flow at high Reynolds number, where the small-scale motion near the wall may determine flow separation. A very fine grid would be needed to capture the small-scale accelerating flow around the sharp corner and the laminar sublayer of the separating boundary layers. At such resolutions, LES of flow in urban canopies would not be feasible. Nevertheless, the flow speedup outside the near-wall region and the location of peak speed-up is well reproduced already by the G2 simulation.

In summary, the grid resolution study illustrates that the medium grid G2 simulation (6 grid points across the bluff body) with the Lagrangian dynamic model is reasonably close to the fine grid simulation (8 grid points across the bluff body), indicating that it can be used as a minimum resolution.

Next, the various subgrid model performances are analyzed. Mean velocities from three different SGS models (not shown) using the G3 grid are compared and show few differences. Substantial differences among the models become apparent when snapshots of eddy-viscosity are examined and model coefficients are compared. The eddyviscosity using SM (Figure 5(a) shows a snapshot of the distribution of eddy-viscosity) is large in the wake region but also in the front shear layers where the flow is expected to be laminar and no eddy-viscosity should be applied. This deficiency of the traditional Smagorinsky model arises because it is based on the strain-rate magnitude that can be large in strongly strained laminar flow. Conversely, the 


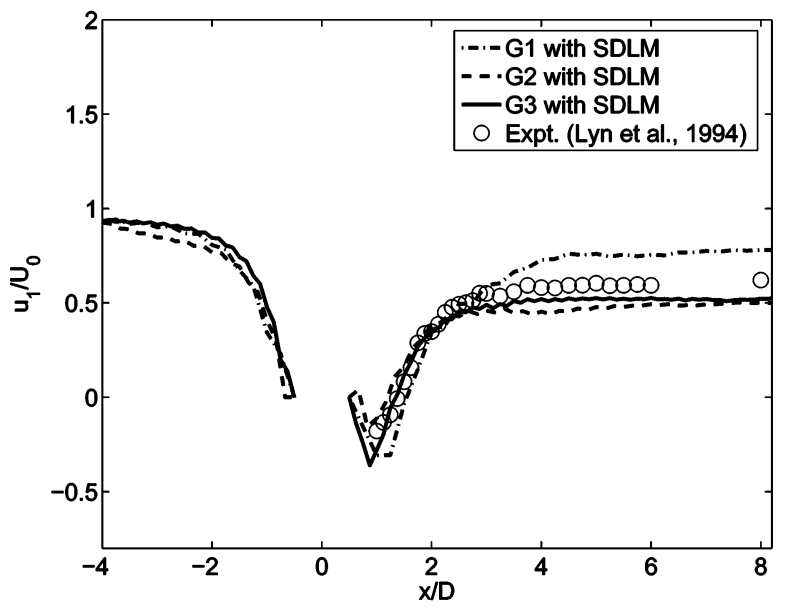

(a)

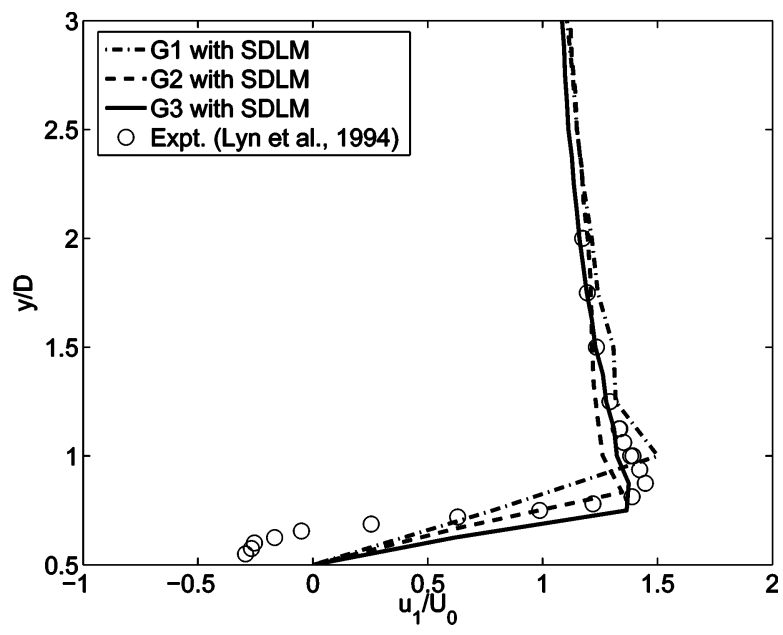

(b)

FIGURE 4. Comparison of mean streamwise velocity $\left(u_{1}\right)$ on different grids (a) along the wake centerline $(y / D=0)$ and $(b)$ in the boundary layer at the center $(x / D=0)$ of the cylinder side surface.

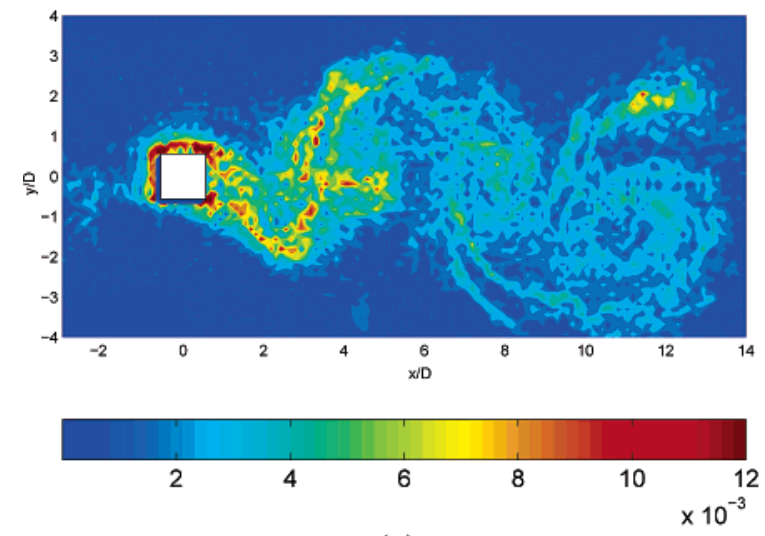

(a)

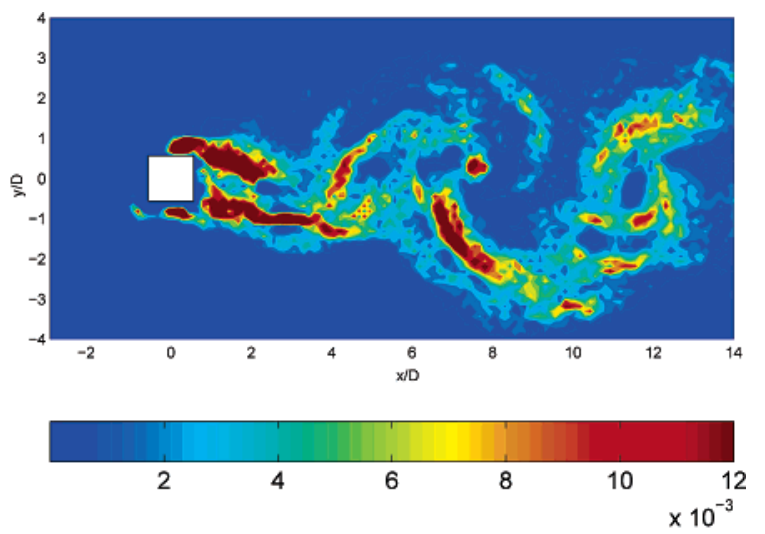

(b)

FIGURE 5. Instantaneous contours of the SGS viscosity using SM (a) and SILM (b) in G3.

Lagrangian dynamic models give a physically more realistic SGS viscosity field, with large values only near the side boundary layers and in the wake. Quantitatively, the maximum values occur in very high shear regions. In general, these maximum values are larger than the eddy-viscosity in the SM in these regions. However, in most other regions, the Lagrangian model produces smaller values.

Inspection of instantaneous contours of SGS coefficient $c_{\mathrm{s}, \Delta}$ for SILM and SDLM (not shown) confirms that, in general, the SDLM results produce larger $c_{\mathrm{s}, \Delta}$ than the SILM results, generally increasing the dissipation of the resolved flow and consequently dampening the resolved fluctuations in SDLM compared to SILM.

Figure 6 shows temporal frequency spectra for the crossflow velocity on the centerline of the wake. About 16000 samples at downstream location $x / D=1$ of velocities are collected over a time interval $T U_{0} / D \sim 20$ (with $50 \%$ overlapping). The spectra calculated from these time series are averaged at three vertically adjacent points to increase the statistical sample. The frequency is nondimensionalized by $U_{0} / D$. The results have peaks at the shedding frequency. As expected, Figure 6 shows that more energy is resolved in the high-resolution G3 case than in the G2 case. The current simulations also reproduce parts of an inertial subrange (the dashed line (- -) shown in Figure 6 has a slope of -5/3). Consistent with results from ref 19 , the SILM yields coefficients that are unrealistically small due to the assumption of scale-invariance, and hence underestimates kinetic energy dissipation, leading to a "pile-up" of energy in the spectra.

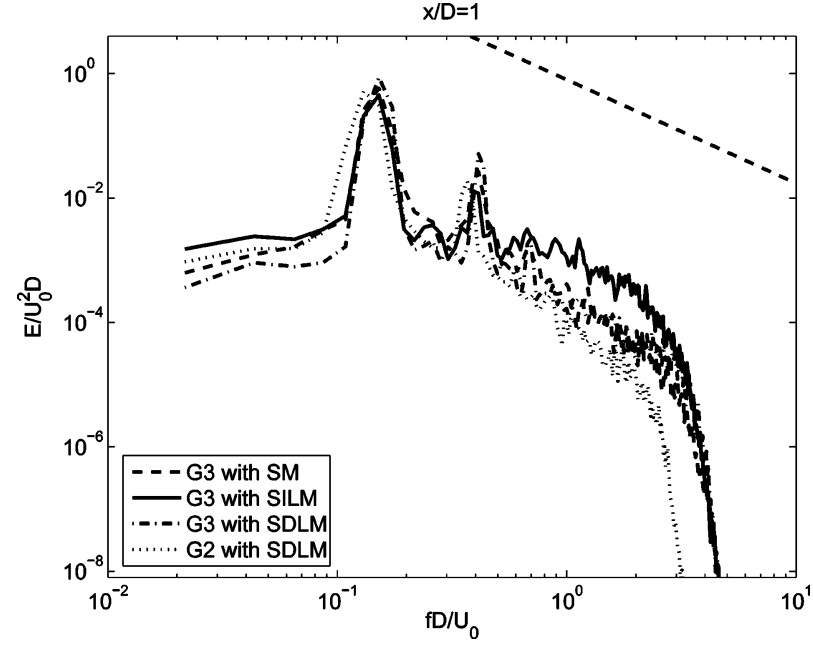

FIGURE 6. Comparison of one-dimensional frequency spectra for spanwise velocity component $\left(u_{2}\right)$ at downstream $x / D=1$ on $G 3$ grid using different models and on $G 2$ grid with the SDLM. The dashed line "- -" has a slope of $-5 / 3$.

The SDLM, however, shows a clear $-5 / 3$ spectrum and thus no unphysical pile-up of energy. In summary, these results show that G2 using the SDLM yields acceptable results in terms of mean velocity distributions and realistically models the physical properties of the eddy-viscosity and energy spectra. 


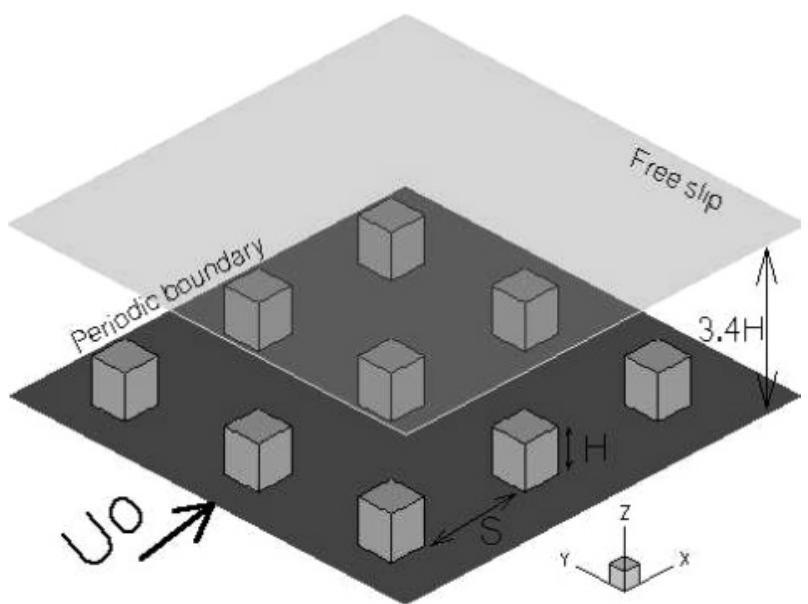

FIGURE 7. Computational domain of flow over periodic square cylinder.

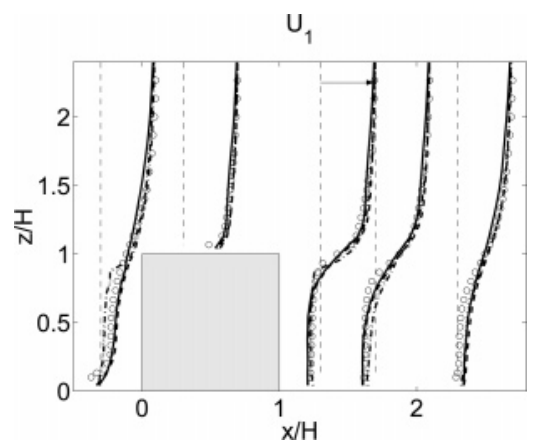

FIGURE 8. Comparison of time-averaged streamwise velocity along the center plan through the cube $(y / H=0)$. The vertical profiles are compared with different SGS models on grids GR1 and GR2 (- • GR1 with SM; -.. GR1 with SDLM; - - GR2 with SM; - GR2 with SDLM; $O$ : experiment). The arrow at the reference location denotes the reference velocity $u_{r}$.

\section{Three-Dimensional Flow over a Matrix of Surface-Mounted Cubes}

The second test case is turbulent flow over a matrix of surfacemounted cubes involving more complex vortical structures. This flow was investigated experimentally by Meinders and Hanjalić $(37,8)$, who made detailed measurements of the mean flow and turbulence using a two-component laser Doppler anemometer (LDA) system within an extensive array of cubes immersed in a two-dimensional channel flow. Two hundred fifty cubes of side length $H$ were placed in an aligned configuration consisting of 25 rows of 10 cubes each with cube spacings of $3 \mathrm{H}$ in both streamwise and spanwise directions. The depth of the plane channel is $3.4 H$. They made measurements at five locations in a unit cell around the 18th row of the array. For these locations, a fully developed and periodic flow is achieved, and the influences of both inflow and outflow boundary conditions are negligible. The geometry of the surface-mounted cubes array is shown in Figure 7. To simulate the flow in this setup, we use a computational domain consisting of an array of nine cubes. This subchannel unit has dimensions of $12 \mathrm{H} \times 12 \mathrm{H} \times 3.4 \mathrm{H}$. The periodic boundary condition is used in both streamwise and spanwise direction. At the bottom boundary and the cube surfaces, the log-law and impermeability conditions are used. The free-slip boundary condition is used at the top. Two computational grids (GR1: $72 \times 72 \times 40$ and GR2: 96 $\times 96 \times 40$ nodes) are used to show that reasonably accurate results are obtained using both these resolutions. The grid is equally spaced and has minimum grid spacing of about $0.167 \mathrm{H}$ (6 grid points across the cube) in GR1 and $0.125 H$ (8 grid points across the cube) in GR2, respectively. A grid sensitivity study was conducted using a finer grid of $144 \times$ $144 \times 40$. We concentrate on the GR1 results because it corresponds to what will be practical in ABL simulations of buildings in urban canopies. The profiles of mean velocity are obtained by time-averaging. After carrying out the simulation for several large eddy turnover times $\left(T_{\mathrm{t}}=H / U_{0}\right.$, $U_{0}$ is the mean bulk velocity) to ensure that final timeaveraged results were independent of the initial conditions, we averaged the instantaneous velocity over 80000 time steps (corresponding to an averaging time of $50 T_{\mathrm{t}}$ ).

The streamwise velocity profiles obtained by the LES with two different SGS models are compared to the experimental data from ref 37 at various downstream distances. Because in prior validation studies we found the SILM results very similar to SDLM results, and because SILM produces larger errors when simulating simple homogeneous surface ABL flow, we focus on the comparison between SM and SDLM models. Figure 8 shows the vertical profiles of the mean streamwise velocity in the vertical center plane $y / H=0$ for the cube from five selected $x$-locations (at $x / H=-0.3,0.3$, $1.3,1.7$, and 2.3 ). The predicted mean streamwise velocity agrees reasonably well with the experimental results. Small discrepancies occur in the recirculation region behind the cubes on coarse grid GR1. Figure 9 shows the horizontal profiles of the mean streamwise and spanwise velocity at $z / H=0.5$ (half cube height) at the same locations. The origin of the coordinate system is located at the upstream corner of the center cube, see Figure 8. All velocities are normalized by a reference velocity, $u_{\mathrm{r}}$, taken as the velocity sufficiently above the cubes, at a height $z / H=2.25$ and at a downstream location of $x / H=1.3$. In Figure 9(a), the flow between cubes in the spanwise direction $(y / H>0.5)$ is unidirectional, while

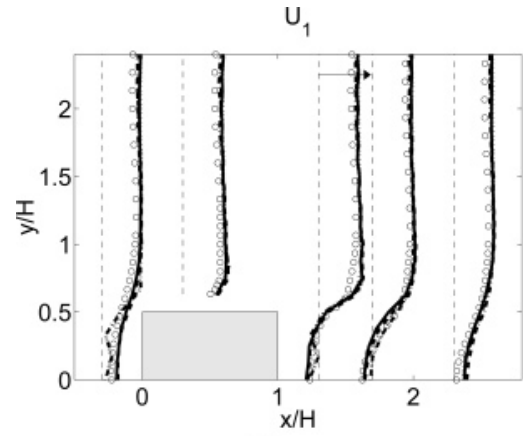

(a)

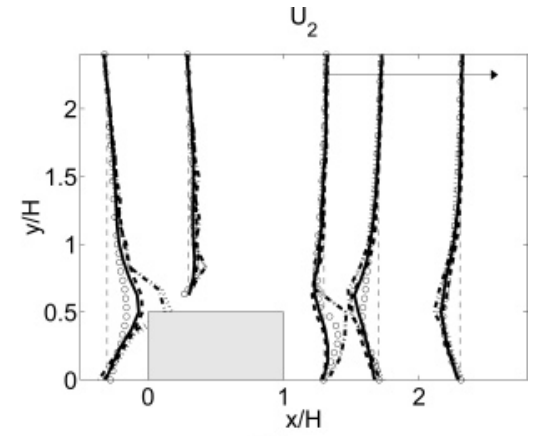

(b)

FIGURE 9. Comparison of the time-averaged (a) streamwise velocity and (b) spanwise velocity with different SGS models along the horizontal plane at half cube height $(z / H=0.5)$ at grids GR1 and GR2 (- - GR1 with SM; $\cdots$ GR1 with SDLM; - - GR2 with SM; - GR2 with SDLM; O: experiment). The arrow at the top denotes the reference velocity $u_{r}$. 

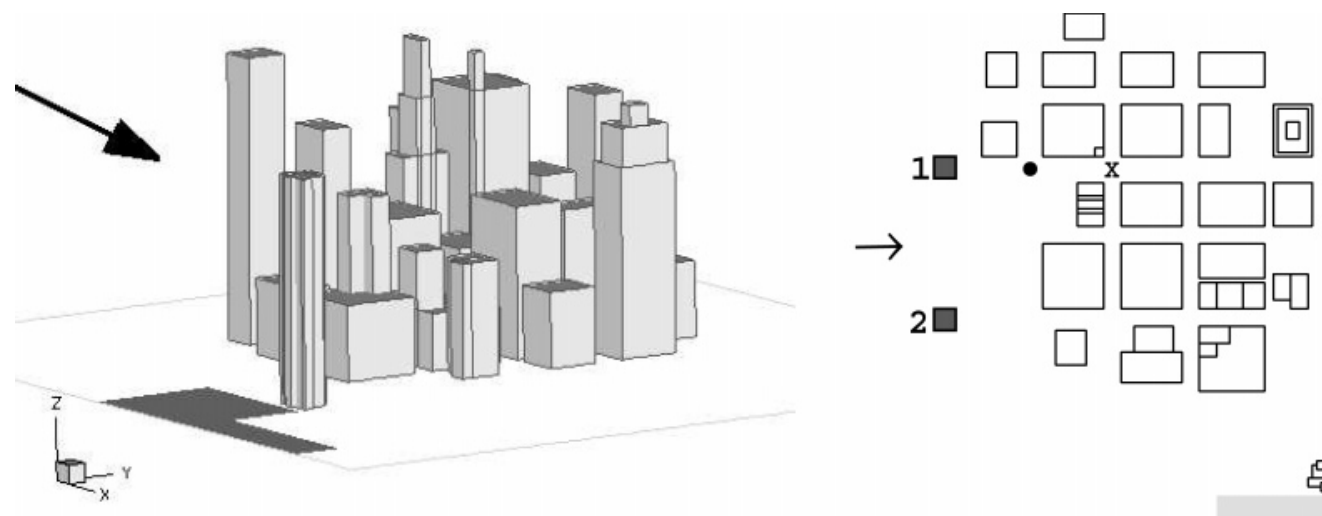

(a)

(b)

FIGURE 10. (a) Skyline and group of buildings in downtown Baltimore, MD. (b) Simulation domain and the locations (1 and 2) of pollutant sources. Shaded region at the bottom represents the water in the Inner Harbor (parametrized by different roughness). The pollutant concentration is observed at various downstream locations from the source (results presented for observer position $x_{1}$ indicated by $\bullet$.

the wake flow behind the cube is reversed. Overall, the agreement between the LES results and the experiments is fairly good, except for the GR1 results at the location $x / H=$ -0.3 (Figure 9 (b)). There, around the front corner of the cube, the computed vertical velocity greatly exceeds the measurement data. The disagreement is due to insufficient grid resolution. The velocity vector at the first grid point in front of the cube would have to turn by nearly $90^{\circ}$ to satisfy the logarithmic tangential and zero normal velocity boundary condition on the cube. The finer G2 grid shows substantially better agreement and convergence. In terms of subgrid closure, the Smagorinsky and SDLM models perform reasonably well in the present tests in neutrally stratified flow. Because the energy spectra appear to be better predicted by SDLM, and also the SDLM is able to reproduce quite well the effects of stratification and buoyancy as shown in other studies (38), we choose to use SDLM in simulations of urban canopy flows in the next section.

\section{Application to Flow and Transport through Downtown Baltimore, MD}

To study the flow and pollutant transport in a realistic setting, we use the LES code to simulate the flow in downtown Baltimore, MD, a complex configuration of several tall buildings. The domain boundaries used in the simulation are shown in Figure 10. Each building's location, size, and height are obtained from a geographic information systems (GIS) database.

The computational domain is $0.6 \mathrm{~km} \times 0.6 \mathrm{~km} \times 0.4 \mathrm{~km}$ $(L \times L \times H)$ represented by $160 \times 160 \times 40$ nodes. We use our results from the preceding section that demonstrated that buildings can be reasonably resolved using 6-8 grid points, as a guideline for the current urban-scale study.

Annual statistics indicate prevailing easterly wind directions, and, accordingly, we force the turbulent inflow $\left(U_{\text {in }}\right)$ from the west, where it is imposed at each time step. This inflow velocity field on the inlet plane is obtained from a separate flow simulation without buildings that uses periodic boundary conditions in the streamwise direction and an imposed friction velocity (imposed pressure drop) of $u^{*}=$ $0.45 \mathrm{~m} / \mathrm{s}$. A flow cycle of 2000 time steps is used. The turbulent inflow in the last five time steps is interpolated from the inflow at the initial and final time steps to filter out the possible jump in the time signals.

The boundary condition along the building surfaces is the standard wall-function that specifies logarithmic conditions (described in section 2). In addition, we parametrize the effects of water in the Inner Harbor using a smaller surface roughness (5), indicated by the shaded regions shown in Figure 10. The roughness used in the domain surfaces is $z_{0}$ $=0.1 \mathrm{~m}$, while it is $z_{0}=0.01 \mathrm{~m}$ on the nodes corresponding to the (smoother) water surface.

Pollutants or scalar particulates are "released" using an imposed scalar flux boundary condition from locations 1 and 2, shown in Figure 10(b). The imposed flux is $Q=0.06$ $\mathrm{g} / \mathrm{m}^{2} \mathrm{~s}$. The pollutants are transported downstream and detour around the building structures within downtown Baltimore. Figure 11 shows two isosurfaces of the pollutant concentration $\left(C / C_{0}=0.002,0.0015\right)$ at time $t=1.5 T_{0}$, where $T_{0}$ is defined as $L / \bar{U}_{\text {in }}$. Concentration is normalized by $C_{0}=$ $Q / u^{*}$, where $u^{*}$ is the inlet friction velocity $(0.45 \mathrm{~m} / \mathrm{s})$ and $Q$ is the imposed flux from the source. Two plumes, one emitted from point 1 and the other from point 2, are first deflected and blocked by the buildings. The plumes are then channeled within the street "canyons". Once the plumes get above the lower rooftop, they travel eastward with the prevailing wind. It is clear that the dispersion depends strongly on the local arrangement of the buildings.

The complex arrangement of buildings influences the flow pattern, resulting in local ground-level winds in the upstream direction. For idealized street canyon simulations, the direction and strength of the channeled wind is often assumed to be proportional to the wind aloft parallel to the street canyon. For complicated building arrangements, however, these assumptions may not hold. Tall buildings can substantially deflect flows, and simple rules for channeling may not be appropriate (39).

For hazardous contaminant applications, we are not only interested in reasonable estimates of the mean concentration but also in the probability of intense events because even low values of concentration may be of serious concern. The temporal variation and Probability Density Function (PDF) distribution at point $x_{1}$, downstream of location 1 (see Figure 10 for locations), are shown in Figure 12. Concentration is measured at three heights, namely $z=5,10,20 \mathrm{~m}$. The simulated pollutant concentration shows relatively strong fluctuations. This is consistent with many field experiments that have found that near-source dispersion and mixing is enhanced in urban areas (40). Wind tunnel experiments have also shown that buildings significantly impact both lateral and vertical dispersion close to the source and immediately downwind (41). The nature of increased turbulent mixing depends strongly on the geometry of the buildings and their 


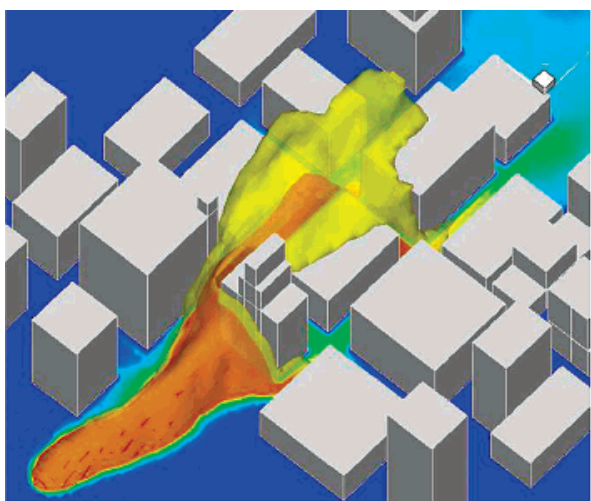

(a)

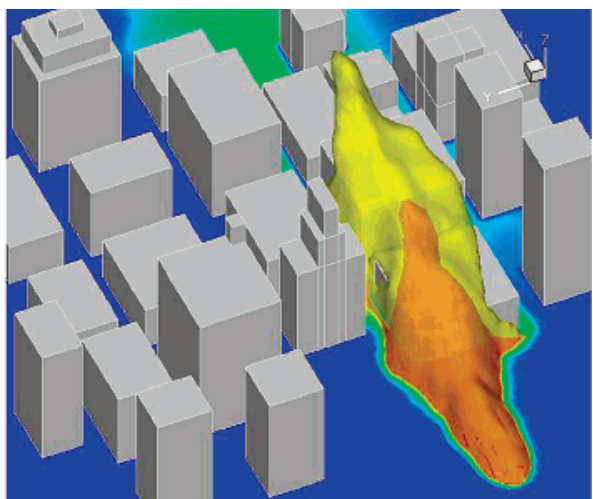

(b)

FIGURE 11. Isosurfaces of the pollutant concentration $\left(C / C_{0}=0.002,0.0015\right)$ released from two different locations across the downtown Baltimore buildings layout. In (a) the release is from location 1, in (b) it is from location 2 . The vertical scale has been reduced to facilitate visibility. On the bottom surface, concentrations are indicated such that maximum concentration in the domain is represented by red, while blue represents zero concentration.

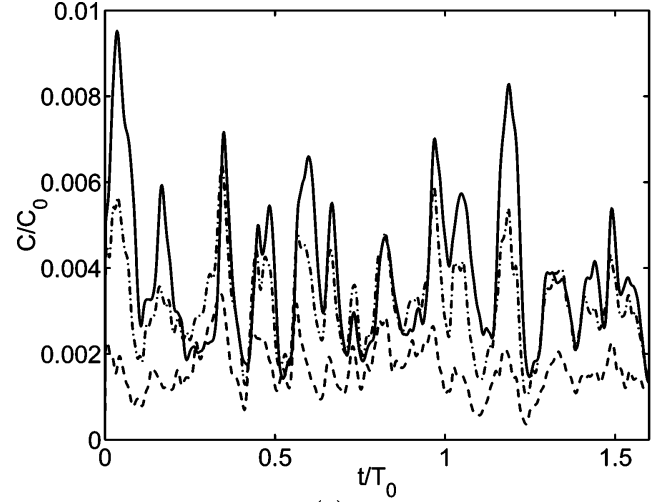

(a)

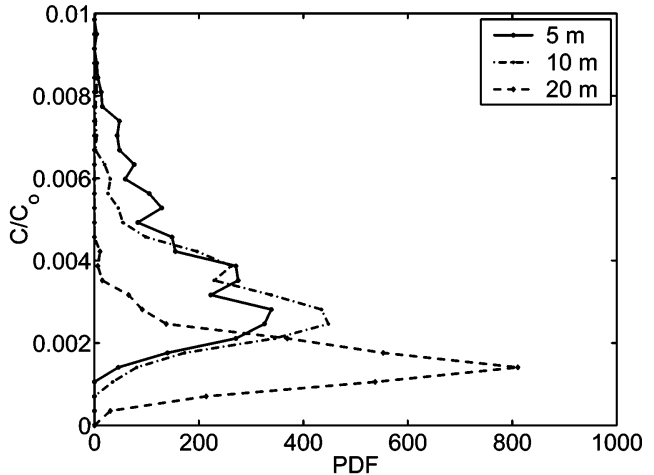

(b)

FIGURE 12. (a) Temporal variations and (b) PDF distribution of the pollutant concentration field $C / C_{0}$. Concentration is observed in downstream location $X_{1}$.

layout, factors that are difficult to parametrize through plume spread curves in traditional Gaussian plume models.

These results show that using LES, turbulent mixing can be analyzed and quantified directly based on the PDFs (see refs 42 and 43 for other applications of PDFs determined from LES), although it must be mentioned that the accuracy of LES in predicting extreme events has not yet been studied in detail. For instance, unresolved scalar fluctuations might affect the tails of the PDFs. More research is required to formulate subgrid models that may be used to augment PDFs computed from LES. In terms of computational cost, LES remains fairly expensive when compared to traditional approaches. In the present simulations of urban dispersion, the total time to simulate a flow time of $4.2 T_{0}$ took about 130 h clock time on a dedicated Intel Xeon $2.8 \mathrm{GHz}$ dual-processor PC.

It is important that LES tools be validated in flows where precise experimental data are available. Our validation-LES of two laboratory experiments used grid resolutions comparable to those that can be applied in LES of ABL flow over urban canopy, where individual buildings are resolved with 6-8 grid-points across the building. Different eddy-viscosity models were tested, including the Lagrangian scale-dependent dynamic model. The latter was found in prior work (19, 18) to be required for proper representation of SGS turbulence without adjustable coefficients while still blending correctly to the log-law near the ground. In the two validation cases, the effects of different SGS models on the mean flow were found to be minor, but we observed that the dynamic Lagrangian models give a physically more realistic SGS viscosity field that vanishes in laminar portions in front of the bluff body when a laminar inflow is present. With these results as guidance, the LES code with the Lagrangian scaledependent dynamic model was applied with increased confidence to atmospheric flow over an urban canopy with realistic boundary conditions corresponding to downtown Baltimore. In general, while LES requires significantly more computer resources than traditional models, the quantity and quality of the results are indisputably superior. In particular, LES provides far more detailed information about the relevant physical transport processes. For example, it allows us to determine probability density functions and probabilities of extreme events directly from the simulated fields and their time dependence.

\section{Acknowledgments}

This research was funded by the EPA Center for Hazardous Substances in Urban Environments (Grant R828771-0-01). We thank the Baltimore City Mayor's Office of Information Technology for providing the GIS database of downtown Baltimore. We also thank Vijayant Kumar, Stuart Chester, and Elie Bou-Zeid for their contributions to recent developments of the JHU-LES code.

\section{Literature Cited}

(1) Kastner-Klein, P.; Plate, E. J. Wind-tunnel study of concentration in street canyons. Atmos. Environ. 1999, 33, 3973-3979.

(2) Rafailidis, S. Influence of building areal density and roof shape on the wind characteristics above a town. Boundary-Layer Meteorol. 1997, 85, 255-271.

(3) Brown, A. R.; Hobson, J. M.; Wood, N. Large-eddy simulation of neutral turbulent flow over rough sinusoidal ridges. BoundaryLayer Meteorol. 2000, 98, 411-441. 
(4) Lien, F. S.; Yee, E. Numerical modelling of the turbulent flow developing within and over a 3-d building array, Part I: a highresolution Reynolds-Averaged Navier-Stokes approach. Boundary-Layer Meteorol. 2004, 112, 427-466.

(5) Bou-Zeid, E.; Meneveau, C.; Parlange, M. B. Large-eddy simulation of neutral atmospheric boundary layer flow over heterogeneous surfaces: Blending height and effective surface roughness. Water Resour. Res. 2004, 40, W02505.

(6) Patnaik, G.; Boris, J. P.; Grinstein, F. F.; Iselin, J. P. In Highresolution schemes for convection-dominated flows: 30 years of FCT; Springer, 2005; pp 105-130.

(7) Lyn, D. A.; Rodi, W. The flapping shear layer formed by flow separation from the forward corner of a square cylinder. J. Fluid Mech. 1994, 267, 353-376.

(8) Meinders, E. R.; Hanjalić, K. Vortex structure and heat transfer in turbulent flow over a wall-mounted matrix of cubes. J. Heat Fluid Flow 1999, 20, 255-267.

(9) Sohankar, A.; Davidson, L.; Norberg, C. Large eddy simulation of flow past a square cylinder: Comparison of different subgrid scale models. ASME J. Fluids Eng. 2000, 122, 39-47.

(10) Nakayama, A.; Vengadesan, S. N. On the influence of numerical schemes and subgrid-stress models on large eddy simulation of turbulent flow past a square cylinder. Int. J. Numer. Methods Fluids 2002, 38, 227-253.

(11) Tseng, Y. H.; Ferziger, J. H. A ghost-cell immersed boundary method for flow in complex geometry. J. Comput. Phys. 2003, 192, 593-623.

(12) Tseng, Y. H.; Ferziger, J. H. Large-eddy simulation of turbulent wavy boundary flow - illustration of vortex dynamics. J. Turbul. 2004, 5, 34.

(13) Smagorinsky, J. General circulation experiments with the primitive equations, I. The basic experiment. Monthly Weather Rev. 1963, 91, 99-164.

(14) Lilly, D. K. In Proceedings of IBM Scientific Computing Symposium of Environmental Sciences; IBM Data Process. Div.: Whiteplains, NY, 1967; p 195.

(15) Germano, M.; Piomelli, U.; Moin, P.; Cabot, W. H. A dynamic sub-grid scale eddy viscosity model. Phys. Fluids 1991, A(3), 1760-1765.

(16) Meneveau, C.; Katz, J. Scale-invariance and turbulence models for large-eddy simulation. Annu. Rev. Fluid Mech. 2000, 32, $1-32$.

(17) Meneveau, C.; Lund, T. S.; Cabot, W. H. A Lagrangian dynamic subgrid-scale model of turbulence. J. Fluid Mech. 1996, 319, $353-385$

(18) Bou-Zeid, E.; Meneveau, C.; Parlange, M. B. A scale-dependent lagrangian dynamic model for large-eddy simulation of complex turbulent flow. Phys. Fluids 2005, 17, 025105

(19) Porté-Agel, F.; Meneveau, C.; Parlange, M. B. A scale-dependent dynamic model for large-eddy simulation: application to a neutral atmospheric boundary layer. J. Fluid Mech. 2000, 415, $261-284$.

(20) Orszag, S. A. Numerical computation of turbulent shear flows. Adv. Geophys. 1974, 18, 224-236.

(21) Mason, P. J. Large-eddy simulation-A critical review of the technique. Q. J. R. Meteorol. Soc. 1994, 120, 1-26.

(22) Piomelli, U.; Balaras, E. Wall-layer models for large-eddy simulations. Annu. Rev. Fluid Mech. 2002, 34, 349-374.

(23) Mason, P. J.; Thomson, D. J. Stochastic backscatter in largeeddy simulations boundary layers. J. Fluid Mech. 1992, 242, $51-78$.

(24) Lilly, D. K. A proposed modification of the Germano subgrid scale closure method. Phys. Fluids 1992, A(4), 633-635.

(25) Albertson, J. D.; Parlange, M. B. Surface length-scales and shear stress: implications for land-atmosphere interaction over complex terrain. Water Resour. Res. 1999, 35, 2121-2132.

(26) Cai, W.; Gottlieb, D.; Shu, C. W. Essentially nonoscillatory spectral Fourier methods for shock wave calculations. Math. Comput. 1989, 186, 389-410.

(27) Geer, J.; Banerjee, N. S. Exponentially accurate approximations to piece-wise smooth periodic functions. J. Sci. Comput. 1997, $12,253-287$.

(28) Moeng, C. H. A large-eddy simulation model for the study of planetary boundary-layer turbulence. J. Atmos. Sci. 1984, 41, 2052-2062.

(29) Spalart, P. R. Strategies for turbulence modelling and simulations. Int. J. Heat Fluid Flow 2000, 21, 252-263.

(30) Nikitin, N. V.; Nicoud, F.; Wasistho, B.; Squires, K. D.; Spalart, P. R. An approach to wall modeling in large-eddy simulations. Phys. Fluids 2000, 12, 1629-1632.

(31) Porté-Agel, F. A scale-dependent dynamic model for scalar transport in large-eddy simulations of the atmospheric boundary layer. Boundary-Layer Meteorol. 2004, 112, 81-105.

(32) McDonald, B. E. Flux-corrected pseudospectral method for scalar hyperbolic conservation laws. J. Comput. Phys. 1989, 82, 413-428.

(33) Lyn, D. A.; Einav, S.; Rodi, W.; Park, J. H. A laser-Dopper velocimetry study of ensemble-averaged characteristics of the turbulent near wake of a square cylinder. J. Fluid Mech. 1995, 304, 285-319.

(34) Rodi, W.; Ferziger, J. H.; Breuer, M.; Pourquie, M. Status of large eddy simulation results of a workshop. ASMEJ. Fluids Eng. 1997, 119, 248-262.

(35) Fureby, C.; Tabor, G.; Weller, H.; Gosman, A. Large eddy simulation of the flow around a square prism. AIAAJ. 2000, 38 $442-452$

(36) Camarri, S.; Salvetti, M. V.; Koobus, B.; Dervieux, A. Large-eddy simulation of a bluff-body flow on unstructured grid. Int. J. Numer. Methods Fluids 2002, 40, 1431-1460.

(37) Meinders, E. R. Experimental study of heat transfer in turbulen flows over wall-mounted cubes, Ph.D. Thesis, Delft University of Technology, Delft, The Netherlands, 1998.

(38) Kleissl, J.; Kumar, V.; Meneveau, C.; Parlange, M. B. Numerica study of dynamic Smagorinsky models in Large Eddy Simulation of the atmospheric boundary layer: validation in stable and unstable conditions. Water Resour. Res. 2005, submitted for publication.

(39) Brown, M. J. Urban dispersion challenges for fast response modeling. 5th AMS Urban Environmental Conference; Vancouver, BC, 2004; 13 pp.

(40) McElroy, J. L. Diffusion from low-level urban sources: reexamination using recently available experimental data. J. Appl. Meteor. 1997, 36, 1027-1030.

(41) Davidson, M. J.; Mylne, K. R.; Jones, C. D.; Phillips, J. C.; Perkins, R. J.; Fung, J. C. H.; Hunt, J. C. R. Plume dispersion through large groups of obstacles - a field investigation. Atmos. Environ. 1995, 29, 3245-3256.

(42) Tseng, Y. H.; Ferziger, J. H. Mixing and available potential energy in stratified flows. Phys. Fluids 2001, 13, 1281-1293.

(43) Tseng, Y. H.; Ferziger, J. H. Effects of coastal geometry and the formation of cyclonic/anti-cyclonic eddies on turbulent mixing in upwelling simulation. J. Turbul. 2001, 2, 014.

Received for review August 28, 2005. Revised manuscript received February 13, 2006. Accepted February 24, 2006.

\section{ES051708M}

